\title{
Educación superior en Tecnología Agropecuaria con enfoque territorial
}

\section{Higher education in agricultural technology with a territorial approach}

\author{
Jorge Alberto Palacio Martínez ${ }^{1}$, María Angustias Hinojo Lucena², Santiago Alonso García ${ }^{3}$ \\ ${ }^{1}$ Politécnico Colombiano Jaime Isaza Cadavid, Colombia (japalacio@elpoli.edu.co) \\ ${ }^{2}$ Universidad de Granada, España (marianhl@ugr.es) \\ ${ }^{3}$ Universidad de Sevilla, España (sag@us.es)
}

Recibido el 26 de abril de 2017; revisado el 29 de julio de 2017; aceptado el 15 de agosto de 2017; publicado el 1 de diciembre de 2018

\section{RESUMEN:}

Se realizó un análisis del currículo del programa de Tecnología Agropecuaria del Politécnico Colombiano Jaime Isaza Cadavid, único de este nivel y con esta denominación, acreditado de alta calidad en Colombia. El objetivo fue determinar la pertinencia del programa con el enfoque territorial del desarrollo rural. El enfoque metodológico fue el mixto. Los instrumentos para la obtención de datos cualitativos fueron el análisis documental a partir de la metodología para el análisis curricular propuesta por Posner (2004) y entrevistas dirigidas a las directivas académicas; los datos cuantitativos se obtuvieron mediante la aplicación de un cuestionario a los profesores, estudiantes, egresados y sector empleador. La integración de datos evidenció el desarrollo de un modelo curricular racional, la supremacía de unas áreas de formación sobre las otras, cambios en el perfil de ingreso de estudiantes, incoherencias en el perfil de egreso y un déficit de formación práctica situada en la ruralidad. Se concluye que el programa declara propósitos, contenidos y prácticas pertinentes con el enfoque territorial, que no se desarrollan en la práctica docente.

$\begin{array}{lcr}\text { PALABRAS } & \text { CLAVE: } & \text { EDUCACIÓN } \\ \text { TECNOLÓGICA, } & \text { EDUCACIÓN } & \text { AGRÍCOLA, } \\ \text { REVISIÓN } & \text { CURRICULAR, } & \text { DISEÑO } \\ \text { CURRICULAR, } & \text { EDUCACIÓN } & \text { RURAL, } \\ \text { DESARROLLO RURAL. } & & \end{array}$

A curricular analysis of the Agricultural Technology program of the Politécnico Colombiano Jaime Isaza Cadavid was completed, as this program is the only one of its kind to be accorded a high quality denomination in Colombia. The objective of this analysis was to determine the applicability of this program to its territorial approach on rural development. The methodological focus was mixed. Document review utilizing the methodology for curricular analysis proposed by Posner (2004) was used to collect qualitative data; while interviews directed toward academic directives and quantitative data were obtained through the use of a questionnaire completed by teachers, students, alumni, and business sector. The integration of data from these sources showed the development of a rational curricular model, an emphasis of some areas of study over others, changes in the profiles of matriculating students over time, incoherencies in the profile of graduates, and a lack of practica occurring in rural areas. It was concluded that although the program declares that it has purposes, contents, and practica that are pertinent to a territorial approach, these are not being developed in the instructional practices that are currently being utilized.

KEYWORDS: EDUCATIONAL TECHNOLOGY, AGRICULTURAL EDUCATION, CURRICULUM REVISIONS, CURRICULAR DESIGN, RURAL EDUCATION, RURAL DEVELOPMENT. 


\section{INTRODUCCIÓN}

La educación tecnológica en Colombia es una tipología educativa que se gesta en la sociedad de finales de los años cincuenta y principios de los años sesenta del siglo XX, como una "modalidad de diversificación o de formación «alternativa» a la educación universitaria tradicional" (Gómez, 2015, p. 37). El surgimiento de este nivel de formación en la educación superior en Colombia estuvo inspirado en el cambio educativo y social, la modernización, la división internacional del trabajo y la marginalidad. La expectativa de integrar el país a la modernidad y al progreso técnico, por la vía del desarrollo económico, generaría nuevas ocupaciones y demandas de personal calificado, del cual se esperaba fuera capaz de transferir las nuevas tecnologías. Para la misma época, el contexto económico y social estaba siendo impactado por dinámicas internacionales como la Alianza para el Progreso y el paquete tecnológico de la revolución verde propuesto para el sector agrario. Coincidiendo con estas dinámicas circulaban en América Latina modelos funcionales para el diseño y práctica curricular, "teorías técnicas del curriculum" (Kemmis, 1998, p. 52) y concepciones curriculares, economicistas, tecnocráticas y modernizantes (Galvani, 1990), que derivarían en planes de estudio prescriptivos, concebidos como instrumentos funcionales de la educación, en el contexto de un currículo eficaz. Dentro de estos modelos cabe destacar el de Tyler, "pedagogía pragmática" según (Díaz, 2005, p. 24); "modelo de fábrica de la educación" (Cullen y Hill, 2013, p. 18); “visión tecnológica sistémica" (Díaz, 2003, p. 459), entre los cuales esta autora incluye a Tyler, Taba, Johnson y Mager; por "objetivos conductuales", Tyler y Taba (Casarini, 2012, p. 118) o modelo curricular lineal, de planificación por objetivos, racional, "con orientación al mercado" (Laanemets y Kalamees, 2013, p. 4).

Actualmente, en Colombia la educación superior se imparte en dos niveles: pregrado y posgrado. El nivel de pregrado tiene, a su vez, tres niveles de formación: 1) nivel Técnico Profesional (programas técnicos profesionales); 2) nivel Tecnológico (programas tecnológicos) y 3) nivel Profesional (programas profesionales universitarios). En el año 2015, el Plan Nacional de Desarrollo 2014-2018, creó el Sistema Nacional de Educación Terciaria (SNET), el cual reorganiza la oferta de formación post-media en dos pilares: educación universitaria y profesional (educación técnica). El pilar de Educación Técnica comprende los niveles de técnico superior, especialización técnica y especialización tecnológica. Los programas pertenecientes a este pilar tendrán una clara orientación hacia el mercado laboral, muy similar al modelo español de formación profesional basado en competencias (Hinojo, Cáceres y Raso, 2013) y su diseño curricular se basaría en mapas funcionales sectoriales, normas de competencia laboral y estándares ocupacionales, colocando en un mismo nivel los programas de formación para el trabajo y el desarrollo humano, con los de educación superior. No obstante que el Sistema Nacional de Educación Terciaria ya fue formalizado, las categorías y tipologías que introdujo lo tienen en medio de fuertes tensiones y debates, que hacen dudar del éxito de la propuesta. Cádiz, Villanueva, Astorga y Echenique (2012), plantean que la educación vinculada a la competencia desde un enfoque funcionalista, "podría quedar reducida a una visión materialista” (p. 541), porque solo responde a las "necesidades del mercado" (p. 541). Las demandas que hace la sociedad a las instituciones de educación superior no se pueden reducir exclusivamente a la formación de capital humano para el incremento de la productividad y rentabilidad de ciertos sectores económicos, menos aún en un país como Colombia, donde el medio rural ha sido objeto de un conflicto armado interno por más de cinco décadas y lo que se esperaría de los profesionales del sector agrario sería una importante contribución al logro de una sociedad más humanizada.

Diversos estudios señalan que uno de los problemas que presenta el currículo de los programas del área agropecuaria en Colombia es que declaran propósitos relacionados con la formación integral que finalmente no se cumplen, debido a que la formación se concentra en el saber disciplinar científico-tecnológico, relegando la formación sociohumanística y cultural a un segundo plano. Uno de los aspectos que ha venido agudizando este problema se debe a que la mayoría de los profesores son catedráticos contratados por horas; en consecuencia, las estrategias pedagógicas dominadas por lógicas metropolitanas desplazaron las prácticas docentes situadas en la ruralidad y "basadas en el lugar" (Azano y Stewart, 2015, p. 2), donde ya no concurren la universidad, la empresa y el agricultor (Wang y Liu, 2014), configurando un currículo "desvinculado del entorno y con modelos de expresión y comportamiento ajenos a las referencias culturales propias de los medios rurales" (Ramo, 2011, p. 96) y, por ello, la narrativa dominante en la comunidad estudiantil es que las asignaturas que contribuyen con la formación integral (ética, 
afectiva, comunicativa, estética, ambiental y sociopolítica) no son fundamentales.

En referencia a las ciencias sociales, una Investigación llevada a cabo en Colombia por Méndez (2008), en la Facultad de Ciencias Agropecuarias en la Universidad de Caldas, encontró que era recurrente que profesores y estudiantes respondieran que las asignaturas del área social eran "casi todas de relleno" (p. 4472). Un estudio de gran impacto regional llevado a cabo por Parr, Trexler, Khanna y Battisti (2007), en la Universidad de California, concluye que un diseño de educación agrícola sostenible debe establecer una relación entre agricultura, medio ambiente y comunidad, desde un enfoque interdisciplinario, multidisciplinario, aplicando la teoría en la práctica y relacionándose con los agricultores, de tal forma que el aprendizaje pueda ser "socialmente distribuido" (Hansen, y Greve, 2015, p. 157). En una investigación llevada a cabo por Rodríguez y Ramírez (2015), donde se empleó una metodología "para la formulación participativa de planes de asistencia técnica agropecuaria con enfoque territorial", (p. 323) se concluye que el capital social en la ruralidad se puede fortalecer "facilitando la inserción de las comunidades rurales en instancias de planificación, decisión y evaluación de la asistencia técnica, en el marco de la legislación vigente" (p. 328).

En esa línea argumentativa, es preciso advertir que el contexto social y político del medio rural colombiano experimenta una nueva realidad, matizada por la coyuntura de los acuerdos alcanzados entre el Gobierno y las Fuerzas Armadas Revolucionarias de Colombia (FARC), lo cual ha desencadenado un paquete de políticas públicas y una remozada institucionalidad cuyos objetivos, en el marco del posconflicto, confluyen hacia una apuesta por el desarrollo rural con enfoque territorial. Un elemento para destacar es que tanto la Misión Rural (Ocampo, 2014) como los informes de Desarrollo Humano de las Naciones Unidas para Colombia, se fundamentan en el concepto de desarrollo planteado por Sen, como la expansión de oportunidades, capacidades y libertad (PNUD, 2015). Según Hidalgo (2011), "esta línea de pensamiento, se encuentra enmarcada en la Escuela Alternativa, [...] a la escuela de la modernización" (p. 302). De acuerdo con este autor, la escuela alternativa surge a "mediados de los años cuarenta del siglo XX" (p. 302), siendo su gran aporte, una concepción del desarrollo que se "basa no tanto en el aumento de la riqueza, implícito en las concepciones del desarrollo del resto de las escuelas, sino en la reducción de la pobreza" (p. 302).

En el área del desarrollo territorial, "centrada en la dimensión territorial del desarrollo, con sus múltiples variantes, (desarrollo regional, local, urbano, rural, endógeno, descentralizado, de abajoarriba...)" (p. 304). Hidalgo (2011) destaca, entre otras, las propuestas de desarrollo rural integral de Raanan Weitz (1981), el desarrollo de abajo-arriba de Walter Stöhr y Fraser Taylor (1981), el desarrollo participativo de Orlando Fals Borda (1985), la planificación y gestión estratégica aplicada al desarrollo local de Jeffrey Luke et. al. (1988) y Edward Blakely (1989) y el presupuesto participativo de Tarso Genro y Ubiratán de Souza (1997).

Inspirados en este marco conceptual, el desarrollo rural con enfoque territorial se encuentra actualmente incorporado en la política pública, en la misión rural y en el capítulo "hacia un nuevo campo Colombiano: reforma rural integral", del Acuerdo final para la terminación del conflicto (Acuerdo Final, 2016, p. 12).

Silva y Sandoval (2012) se refieren al territorio, como un espacio "socio-relacional" con una serie de características en común [...]: "una base de recursos naturales específica, una identidad particular, relaciones sociales, institucionales $\mathrm{y}$ formas de organización propias, y determinadas formas de producción, intercambio y distribución del ingreso" (pp. 19-20). Pisani y Francescheti (2011), manifiestan que el enfoque sectorial considera el territorio rural como un área donde se llevan a cabo actividades sectoriales, agrícolas, pecuarias o forestales, mientras el enfoque territorial lo considera una "construcción social" caracterizada por la "interacción entre los distintos actores" (p. 207).

Por su parte, Ambrosio-Albalá y Bastiaensen (2010) identifican que en los procesos territoriales hay que tener en cuenta la transformación productiva e institucional y algunos temas transversales tales como: "1) la innovación; 2) el enfoque integrador y multisectorial (Espíritu empresarial, infraestructura, gobernanza, medio ambiente, así como la relación entre actores sociales de diferentes esferas); y 3 ) la competitividad territorial" (pp. 12-15). En relación con el tema de gobernanza, en el marco del proyecto LEADER (Relaciones entre Actividades de Desarrollo de la Economía Rural) Thuesen y Nielsen (2014) demostraron que el nivel de los grupos de acción local (GAL) mejora el desarrollo local, la democracia participativa y "la toma de decisiones de abajo hacia arriba" (p. 321), con un alto nivel de 
"eficiencia o eficacia" (p. 321). Esto revela el "carácter complejo y multidimensional de las dinámicas territoriales y la influencia decisiva que la disponibilidad de los cinco tipos de capital territorial (económico, humano, social, cultural y ambiental) ejerce sobre ellas" (Agarwal et al., citados en Sánchez, Gallardo y Ceña, 2016, p. 100).

La mayoría de los autores que abordan el enfoque territorial coinciden en que involucra cuatro dimensiones: socio-cultural, económica-productiva, ambiental y político-institucional (Jancovik, 2012; Murcia, 2011). Cada una de ellas busca alcanzar transformaciones relevantes en el territorio: equidad, competitividad, sostenibilidad y gobernanza, respectivamente. Grabowski (2008) anota que las debilidades institucionales en América Latina están asociadas con el grado de desigualdad en el sector rural, agrícola.

Una investigación llevada a cabo por Pachón (2006) sobre las concepciones de desarrollo rural en los estudiantes de una facultad de Agronomía en Bogotá (Colombia) encuentra que, en esta población, existe una tendencia a relacionar el desarrollo rural con aspectos "modernizantes y tecnológicos" (p. 388).

Con base en estos conceptos, el enfoque territorial rural en un escenario de posconflicto, debe propiciar en términos de pertinencia, innovaciones pedagógicas, metodológicas y curriculares en los programas del área agropecuaria, que tienen como escenario de localización laboral de sus graduados el medio rural, lo cual implica, entre otras acciones, desarrollar procesos que fortalezcan la formación integral de los estudiantes. Ahora bien, esto no quiere decir que la atención a demandas relacionadas con la equidad, competitividad, sostenibilidad y gobernanza desplacen la formación científicotecnológico agrícola, pecuaria o agroindustrial, sino que los programas del área amplíen su campo de acción, integrando propósitos, contenidos y prácticas llevadas a cabo en contextos reales con comunidades reales, a través de un compromiso sostenido de las instituciones, estudiantes y docentes con las dinámicas territoriales.

Uno de los aspectos que privilegia el enfoque territorial es justamente un actor social empoderado y protagonista de su propio desarrollo y el de los miembros de su comunidad, como un proceso sinérgico de ampliación de capacidades y libertades, que, en el contexto curricular, debería ser abordado desde un enfoque multidimensional. Varias investigaciones (Sipos, Battisti y Grimm, 2008; Molina, 2010; Wang y Liu, 2014) obtienen resultados similares.
El Politécnico Colombiano Jaime Isaza Cadavid de Medellín, institución en la cual se llevó a cabo esta investigación, fue fundada en 1963 y en 1964 inicia el programa de Tecnología Agropecuaria en Medellín- Colombia.

Sobre la base de los elementos encontrados en la revisión de la literatura, se realizó un análisis del currículo de tecnología agropecuaria, con la participación de los diferentes actores, para identificar la coherencia que existe entre el currículo oficial que declara la formación integral en el marco de una visión multidimensional del desarrollo agropecuario y rural y el enfoque territorial.

\section{MATERIAL Y MÉTODO}

El enfoque metodológico fue el mixto. En la fase cualitativa se llevó a cabo un análisis documental del programa y entrevistas; en la fase cuantitativa un diseño descriptivo, aplicando pruebas de correlación y comparación (tabla 1).

Tabla 1. Ficha técnica de la investigación

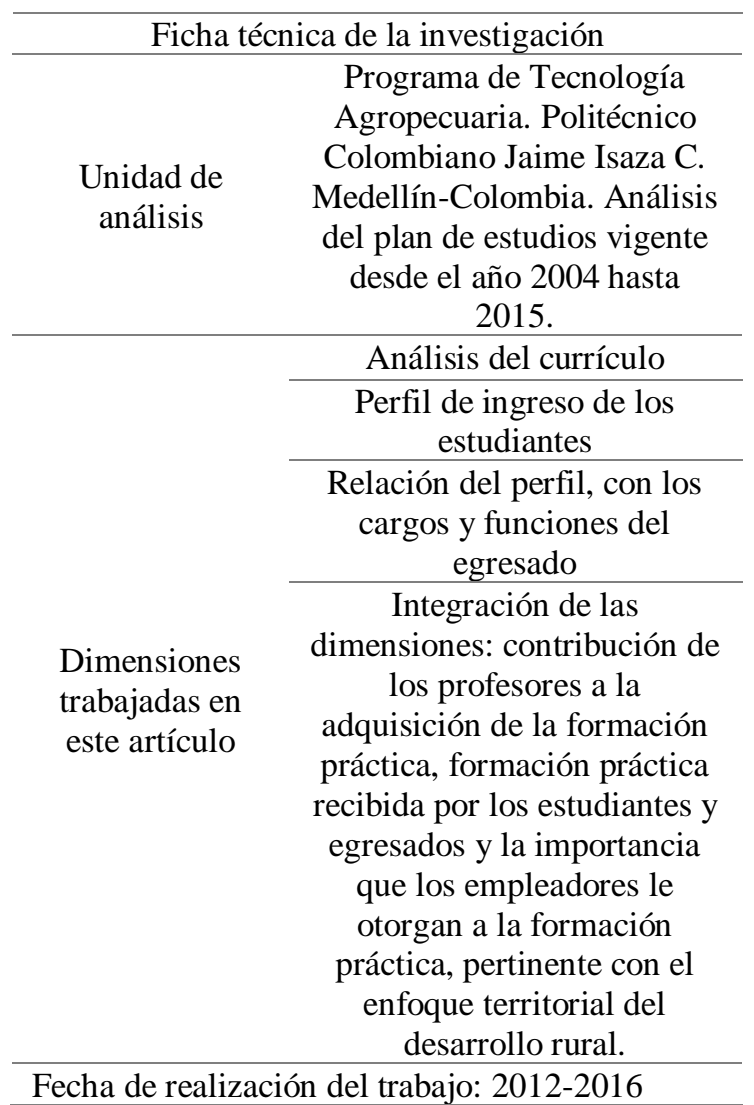

\subsection{Instrumentos para la recolección de datos cualitativos}

Las técnicas empleadas para la recolección de datos fueron, el análisis documental y una entrevista semi- 
estructurada, dirigida al decano y coordinador de la Facultad de Ciencias Agrarias.

Para la dimensión análisis del currículo, se siguió la metodología para el "análisis del curricular" propuesta por Posner (2004), que consiste en el registro, sistematización, análisis y resolución, de un conjunto de 68 preguntas organizadas en cuatro grupos (tabla 2).

Tabla 2. Metodología propuesta por Posner (2004) para el análisis del currículo y la fuente documental

\begin{tabular}{|c|c|c|}
\hline \multicolumn{3}{|c|}{ Dimensión análisis del currículo } \\
\hline Análisis del currículo & $\begin{array}{l}\mathrm{N}^{\circ} \text { de } \\
\text { Preguntas }\end{array}$ & Fue \\
\hline $\begin{array}{l}\text { Primer grupo. Documentación y origen del } \\
\text { currículo }\end{array}$ & & \multirow{15}{*}{$\begin{array}{l}\text { 1) Actas del Consejo de Facultad de } \\
\text { Ciencias Agrarias y del Comité de } \\
\text { Currículo del Programa, durante el } \\
\text { período 2000-2013; 2) Informes de } \\
\text { condiciones de calidad, 2004-2013 para } \\
\text { la renovación de los registros calificados, } \\
\text { por parte del Ministerio de Educación } \\
\text { Nacional; 3) Plantillas de los programas } \\
\text { y guías didácticas de las asignaturas } \\
\text { entre 2004 y 2015; 4) Proyecto } \\
\text { Educativo, 2005 y Modelo Educativo } \\
\text { Institucional 2011; 5) Plan de Desarrollo } \\
\text { Institucional 2010-2016; 6) Informes de } \\
\text { autoevaluación para la Acreditación, } \\
\text { 2006, 2010, 2012; 7) Manual de líneas } \\
\text { de Investigación; 8) Proyectos } \\
\text { Educativos del Programa. 2007 y 2013; } \\
\text { 9) Proyecto Educativo de Facultad 2011; } \\
\text { 10) Tasa de ingreso, graduados y } \\
\text { deserción del programa; y 11) Perfiles de } \\
\text { los docentes vinculados de planta y } \\
\text { catedráticos contratados por horas, entre } \\
\text { otros. }\end{array}$} \\
\hline I. ¿Cómo está documentado el currículo? & 5 & \\
\hline $\begin{array}{r}\text { II ¿Qué situación pro } \\
\text { currí }\end{array}$ & 7 & \\
\hline $\begin{array}{l}\text { III. ¿Qué persp } \\
\text { represent }\end{array}$ & 1 & \\
\hline Segundo grupc & & \\
\hline $\begin{array}{r}\text { IV. ¿Cuáles son los } \\
\text { del } c\end{array}$ & 15 & \\
\hline $\begin{array}{l}\text { V. ¿Qué suposiciones están implícitas en el } \\
\text { enfoque del currículo acerca del propósito o } \\
\text { el contenido? }\end{array}$ & 4 & \\
\hline VI. ¿Cómo está or & 7 & \\
\hline $\begin{array}{l}\text { VII. ¿Cuáles suposiciones están implícitas } \\
\text { en la organización del currículo? }\end{array}$ & 4 & \\
\hline Tercer grupo: El currículo en uso & & \\
\hline $\begin{array}{c}\text { VIII. ¿Cómo debe implementarse el } \\
\text { currículo? }\end{array}$ & 11 & \\
\hline $\begin{array}{l}\text { IX. ¿Qué puede usted aprender del currículo } \\
\text { desde el punto de vista de la evaluación? }\end{array}$ & 10 & \\
\hline Cuarto grupo: Crítica & & \\
\hline X. ¿Cuál es su opinión a & 4 & \\
\hline Total preguntas & 68 & \\
\hline
\end{tabular}

\subsection{Instrumentos para la recolección de datos cuantitativos}

La información se obtuvo a partir de la aplicación de una encuesta tipo cuestionario (tabla 3 ).

Tabla 3. Características del cuestionario, fuentes, tamaño muestral y número de ítems.

Tipo de pregunta de encuesta $\quad$ Se diseñaron cuatro (4) cuestionarios estructurados, dirigidos a los profesores, estudiantes, egresados y empleadores, autoaplicados, tipo likert de respuesta graduada (1 a 4) puntuaciones y de selección, con escala de medición fundamentalmente ordinal.

Fuentes

40 profesores vinculados actualmente de planta y

catedráticos contratados por horas, adscritos parcial o totalmente al Programa.

77 estudiantes activos del plan de estudios vigente, pertenecientes al $4^{\circ}, 5^{\circ}$ y $6^{\circ}$ semestre.

50 egresados del plan de estudios vigente, graduados a partir 2007-01.

19 empleadores de tecnólogos agropecuarios, representativos del medio.
Tamaño muestral

$$
\text { Censo }
$$
$\mathrm{N}^{\circ}$ de Ítems 70

90

$$
\text { Censo }
$$

Muestra no probabilística. No todos los egresados podían ser tenidos en cuenta. Validar el perfil profesional y ocupacional requería experiencia laboral. Muestra no probabilística. Empresas del sector público, 66 privado y solidario, empleadoras de tecnólogos 
Total: 186 individuos

Procesamiento de datos y análisis estadístico cuantitativo. agropecuarios.

Se realizó a través del SPSS Statistics 22.

Para poder establecer correlaciones entre las respuestas dadas por diferentes actores del currículo (profesores, estudiantes, egresados y empleadores) hay tres temas que corresponden a 44 ítems que comparten los cuatro cuestionarios. Este artículo presenta únicamente los resultados relacionados con la contribución de los profesores a la formación práctica, recibida por los estudiantes y egresados, pertinentes con el desarrollo rural con enfoque territorial y la importancia que le atribuye el sector empleador a la formación práctica (tabla 4).

Tabla 4. Formación práctica, número de ítems, fuentes y distribución de ítems.

\begin{tabular}{|c|c|c|c|}
\hline Tema & $\begin{array}{l}\mathrm{N}^{\circ} \mathrm{de} \\
\text { Ítems }\end{array}$ & Fuentes & Distribución de ítems \\
\hline $\begin{array}{l}\text { Formación } \\
\text { práctica }\end{array}$ & 12 & $\begin{array}{l}\text {-El perfil profesional y ocupacional, misión } \\
\text { visión y objetivos del programa } \\
\text {-“Contenidos por unidades" pertinentes con } \\
\text { las cuatro dimensiones del enfoque territorial } \\
\text { del programa vigente, en los formatos } \\
\text { oficiales, "Plantillas del programa y guías } \\
\text { didácticas de la asignaturas", } 2004 \text { a } 2015 \text {. }\end{array}$ & $\begin{array}{l}\text { Del } 1 \text { al 3: dimensión económica-productiva. } \\
\text { Competitividad } \\
\text { Del } 4 \text { al 6: dimensión ambiental. } \\
\text { Sostenibilidad } \\
\text { Del } 7 \text { al 9: dimensión socio-cultural. Equidad, } \\
\text { Del } 10 \text { al 12: dimensión político-institucional. } \\
\text { Gobernanza }\end{array}$ \\
\hline
\end{tabular}

\subsection{Pruebas de validación de cuestionarios y consistencia entre jueces}

Para determinar la fiabilidad y consistencia interna del cuestionario se realizó una validación del contenido de los cuestionarios de profesores, estudiantes, egresados y empleadores, por el método del juicio de expertos. Para determinar la consistencia entre jueces, se empleó el coeficiente W. de Kendall.

\subsection{Correlaciones no paramétricas}

Debido a que se trabajó con datos de tipo ordinal, se utilizaron correlaciones no paramétricas, por lo que se empleó el coeficiente de correlación de Spearman. Para el análisis de comparación por grupos, se aplicó la prueba U de Mann Whitney, la cual resultó útil al comparar 2 muestras independientes. Los grupos para los cuales se aplicó esta prueba fueron: egresados vs estudiantes, profesores vs empleadores y profesores vs estudiantes.

\section{RESULTADOS}

\subsection{Validación de cuestionarios}

Los resultados de la validación de los cuestionarios se muestran en la tabla 5 .
Tabla 5. Estadísticos de fiabilidad de profesores, estudiantes, egresados y empleadores

\begin{tabular}{ccc}
\hline Cuestionario & Prueba & $\mathrm{N}^{\circ}$ de elementos \\
\hline & Alfa de Cronbach \\
\hline Profesores &, 986 & 70 \\
\hline Estudiantes &, 979 & 90 \\
\hline Egresados &, 846 & 80 \\
\hline Empleadores &, 983 & 66 \\
\hline
\end{tabular}

Los resultados arrojaron para todos los casos un índice de confiabilidad alfa de Cronbach superior a 0.8 , índices que aseguran una apropiada consistencia interna.

\subsection{Consistencia entre jueces}

Los resultados de consistencia entre jueces se presentan en la tabla 6.

Tabla 6. Índice de acuerdo interjueces

\begin{tabular}{cccc}
\hline Cuestionario & Prueba & $\begin{array}{c}\mathrm{N}^{\circ} \text { de } \\
\text { elementos }\end{array}$ \\
\hline & $\begin{array}{c}\text { W de } \\
\text { Kendall }\end{array}$ & $\begin{array}{c}\text { Nivel de } \\
\text { significancia }\end{array}$ & \\
\hline Profesores &, 606 & $1,48 \mathrm{E}-05$ & 70 \\
\hline Estudiantes &, 627 & $4,3 \mathrm{E}-05$ & 90 \\
\hline Egresados &, 218 & $3.06 \mathrm{E}-06$ & 80 \\
\hline Empleadores &, 612 & 0,003 & 66 \\
\hline
\end{tabular}

Los resultados indican concordancia significativa entre los rangos asignados por los jueces a cada uno de los criterios establecidos para la valoración del contenido. 
Se presenta a continuación la integración de resultados parciales de las dimensiones mencionadas.

\subsection{Dimensión análisis del currículo}

Los resultados se presentan agrupados en diseño, desarrollo y evaluación curricular.

El diseño curricular. 1) Modelo de currículo racional, siguiendo el modelo para el diseño curricular de Taba H.; 2) Plan de estudios conformado por 34 asignaturas, 96 créditos y cuatro áreas; 3) El porcentaje de créditos asignados a cada área es: básica 21\%; básica tecnológica $23 \%$; profesional específica $47 \%$ y socio-humanística $8.3 \%$, esta última conformada por cinco asignaturas como se muestra en la tabla 7.

Tabla 7. Asignaturas que conforman el área sociohumanística

\begin{tabular}{cccc}
\hline Asignaturas & Nivel & Créditos & Porcentaje \\
\hline Humanidades I & 1 & 2 & $2.1 \%$ \\
\hline Lengua Materna & 1 & 2 & $2.1 \%$ \\
\hline $\begin{array}{c}\text { Pedagogía } \\
\text { Constitucional }\end{array}$ & 2 & 1 & $1.0 \%$ \\
\hline $\begin{array}{c}\text { Deporte, Arte y } \\
\text { Recreación }\end{array}$ & 2 & 1 & $1.0 \%$ \\
\hline Desarrollo Rural & 6 & 2 & $2.1 \%$ \\
\hline Total & & & $8.3 \%$ \\
\hline
\end{tabular}

Desarrollo curricular. 1) El coordinador del programa plantea en la entrevista "La mayoría de egresados y docentes, están inmersos en el sistema tradicional, lo cual se refleja en las bases pedagógicas de cada uno, aquí no hay modelos críticos, es poco el docente crítico, hay docentes constructivistas que terminan siendo desarrollistas, técnicos, positivistas y con enfoque tradicional". El decano expresa "Cada docente hace un desarrollo muy específico desde su saber y experiencia, desde lo que concibe que deba necesitar ese profesional"; 2) propósitos y contenidos de las asignaturas, que no establecen una relación explícita con el perfil de egreso; 3) objetivos de aprendizaje que no trascienden a la ruralidad; 4) estrategias didácticas desarrolladas con fundamento en una epistemología de las ciencias naturales; 5) ambientes de aprendizaje geográficamente dispersos, los salones de clase en la ciudad capital, los laboratorios a 10 $\mathrm{km}$ en un municipio del área metropolitana y las granjas a $40 \mathrm{~km}$ de distancia; 6) el programa se desarrolla exclusivamente en la ciudad capital o en el área metropolitana; 7) programación de asignaturas en horario nocturno, incluido el curso de desarrollo rural; 8) investigación históricamente enmarcada en el paradigma cuantitativo, escaso número de docentes investigadores y bajos indicadores en investigación tecnológica, desarrollo e innovación; y 9) proyectos de extensión rural escasos o inexistentes, formulados por agentes individuales y sin una relación directa con el currículo oficial.

Evaluación curricular. 1) heteroevaluación, con enfoque cuantitativo y por objetivos; 2) participación de los actores del currículo: profesores, estudiantes, egresados y sector empleador, mediada por esporádicos encuentros colectivos, insuficientes para evaluar el currículo y desarrollar una práctica docente que, basada en datos empíricos, articule y le dé coherencia a los objetivos, contenidos, estrategias metodológicas, recursos, medios y procesos evaluativos, con las nuevas dinámicas territoriales.

Cuando a los estudiantes, egresados, profesores y sector empleador se les preguntó, si habían participado o participan con aportes o sugerencias al currículo del programa, el promedio de respuestas por el sí, fue respectivamente: estudiantes un $42 \%$, egresados un $28 \%$, profesores un $55.7 \%$ y empleadores un $21.1 \%$.

Los resultados de los procesos de autoevaluación realizados en 2006, 2010 y 2012, mostraron que de todas las características evaluadas la más baja calificación, la obtiene el impacto de los egresados en el medio social y académico. Tabla 8

Tabla 8. Resultados de los informes de autoevaluación

Característica: impacto de los egresados en el medio social y académico

\begin{tabular}{cccc}
\hline Año & $\begin{array}{c}\text { Valor } \\
\text { porcentual }\end{array}$ & $\begin{array}{c}\text { Valor } \\
\text { asignado } \%\end{array}$ & $\begin{array}{c}\text { Valor } \\
\text { logrado \% }\end{array}$ \\
\hline 2006 & 45.0 & 4.5 & 2.02 \\
\hline 2010 & 43.00 & 2.64 & 1.13 \\
\hline 2012 & 69.95 & 3.75 & 2.62 \\
\hline
\end{tabular}

\subsection{Dimensión perfil de ingreso de los estudiantes}

El desarrollo de esta dimensión contiene datos parciales obtenidos de la aplicación del cuestionario a los estudiantes.

Estudiantes: 1) cuando ingresaron al Programa un $89.6 \%$ de estudiantes matriculados en el $4^{\circ}, 5^{\circ} \mathrm{y}$ $6^{\circ}$ semestre, residía en el Área Metropolitana. 2) un $76.6 \%$ de los estudiantes está totalmente en desacuerdo en haber culminado sus estudios en una Institución Educativa con media técnica en agropecuaria.

Cuando a los estudiantes se les preguntó los motivos por los cuales ingresaron al programa, algunas de las mayores frecuencias de respuesta en las que estuvieron totalmente de acuerdo fueron: 1) 
un $54.5 \% \%$, me gusta trabajar con cultivos agrícolas; 2) un $53.2 \%$, me brinda la posibilidad de trabajar en el campo; 3) un $46.8 \%$, me gusta trabajar con animales domésticos; 4) un 53.2\% está de acuerdo en que le gusta trabajar con comunidades rurales; 5) un $45.5 \%$ está de acuerdo, en que ingresó al programa porque está acreditado de alta calidad y a que su vocación es ser tecnólogo agropecuario, respectivamente.

\subsection{Dimensión relación del perfil con los cargos $y$ funciones del egresado}

Esta dimensión se desarrolló a partir de los datos obtenidos de la aplicación del cuestionario dirigido a los egresados y empleadores.
Egresados: 1) los egresados del programa registran un desempleo del $38 \%$; 2) un $34 \%$ devenga entre 1 y 2 salarios mínimos legales vigentes (smlv) y un 52\% entre 1 y 4 smlv. Esto indica que en 2015 , el $34 \%$ de los egresados devengaba entre 258 y 516 dólares mensuales.

El actual perfil de egreso plantea seis (6) posibles cargos que el tecnólogo agropecuario puede desempeñar y diez (10) posibles funciones. Cuando a los egresados y empleadores se les consultó por los cargos que ocupan y las funciones que realizan, se registran los resultados que se muestran en la figura 1 y tabla 9.

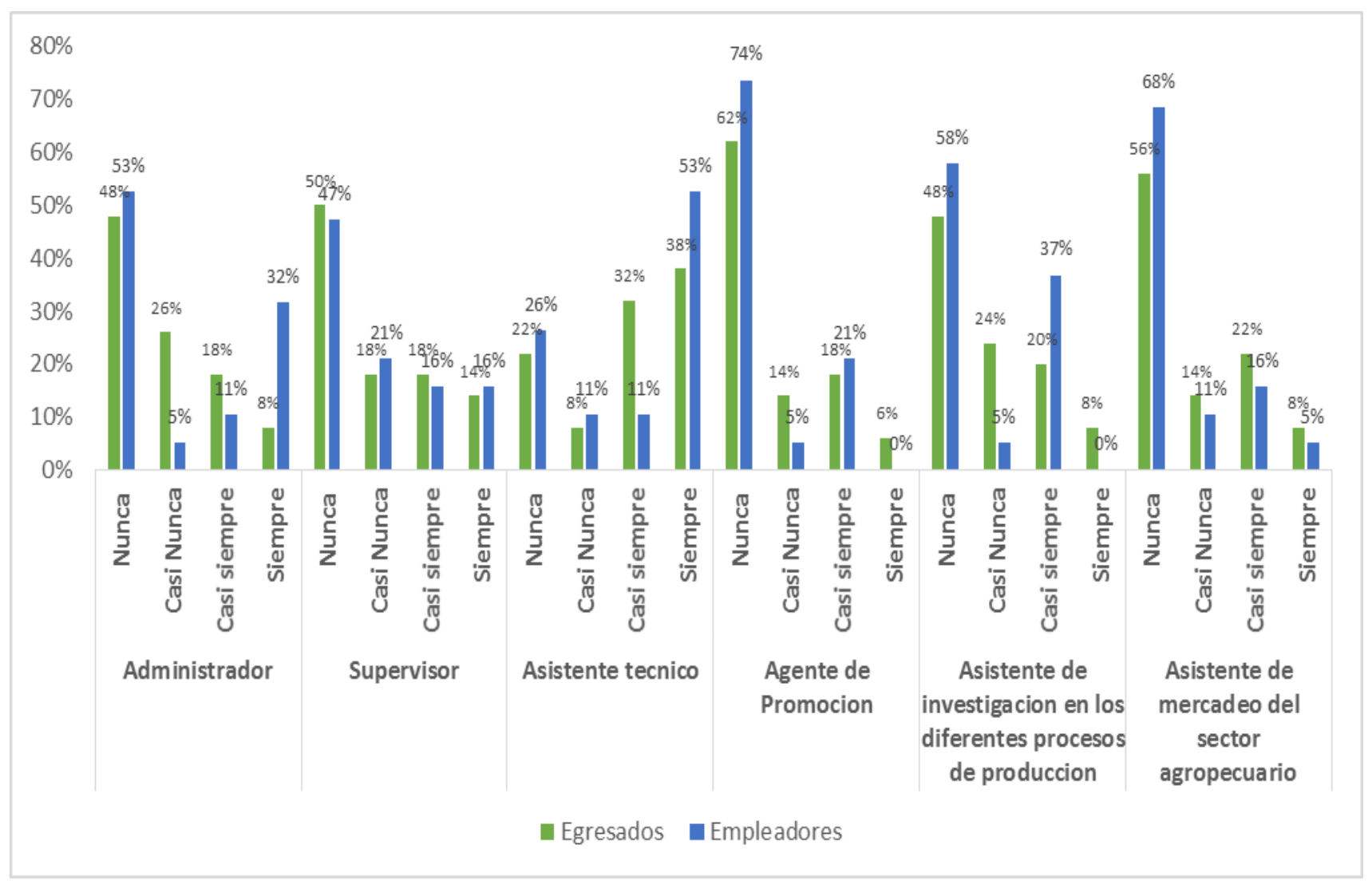

Figura 1. Mayor frecuencia de respuesta con relación al cargo que desempeñan, según egresados y empleadores

Tabla 9. Mayor frecuencia de respuesta con relación a las funciones que realizan, según egresados y empleadores

\begin{tabular}{ccccccc}
\hline Ítem & \multicolumn{3}{c}{ Egresado } & \multicolumn{3}{c}{ Empleador } \\
\cline { 2 - 7 } & Frecuencia & $(\%)$ & Escala & Frecuencia & $(\%)$ & Escala \\
\hline $\begin{array}{c}\text { Administrar programas de } \\
\text { producción en el campo } \\
\text { agropecuario. }\end{array}$ & 18 & 36 & Nunca & 9 & 47,4 & Siempre \\
\hline $\begin{array}{c}\text { Mejorar y conservar la producción } \\
\text { mediante la apropiación de } \\
\text { técnicas y tecnologías. }\end{array}$ & 17 & 34 & Nunca & 12 & 63,2 & Casi \\
siempre \\
\hline $\begin{array}{c}\text { Dirigir programas de conservación } \\
\text { y manejo racional de los recursos }\end{array}$ & 17 & 34 & Siempre & 9 & 47,4 & $\begin{array}{c}\text { Casi } \\
\text { siempre }\end{array}$ \\
\hline
\end{tabular}




\begin{tabular}{|c|c|c|c|c|c|c|}
\hline naturales renovables. & & & & & & \\
\hline $\begin{array}{l}\text { Programar la utilización, } \\
\text { operación y mantenimiento de } \\
\text { maquinaria, implementos y } \\
\text { herramientas. }\end{array}$ & 26 & 52 & Nunca & 7 & 36,8 & Casi nunca \\
\hline $\begin{array}{l}\text { Desarrollar programas de } \\
\text { extensión rural conducentes a la } \\
\text { solución de problemas en la } \\
\text { producción agropecuaria de } \\
\text { pequeños productores. }\end{array}$ & 18 & 36 & Nunca & 9 & 47,4 & $\begin{array}{c}\text { Casi } \\
\text { siempre }\end{array}$ \\
\hline Gestar empresas en el sector & 28 & 56 & Nunca & 7 & 36,8 & Nunca \\
\hline $\begin{array}{l}\text { Planear, dirigir, controlar y evaluar } \\
\text { proyectos agropecuarios. }\end{array}$ & 17 & 34 & Nunca & 8 & 42,1 & $\begin{array}{c}\text { Casi } \\
\text { siempre }\end{array}$ \\
\hline $\begin{array}{c}\text { Participar en grupos de } \\
\text { investigación especializada. }\end{array}$ & 25 & 50 & Nunca & 10 & 52,6 & Nunca \\
\hline $\begin{array}{c}\text { Ejecutar programas de instrucción } \\
\text { agropecuaria en centros o } \\
\text { comunidades. }\end{array}$ & 15 & 30 & Siempre & 8 & 42,1 & Siempre \\
\hline $\begin{array}{c}\text { Desarrollar campañas relacionadas } \\
\text { con el adelanto del sector } \\
\text { campesino }\end{array}$ & 17 & 34 & $\begin{array}{c}\text { Casi } \\
\text { siempre }\end{array}$ & 9 & 47,4 & Siempre \\
\hline
\end{tabular}

\subsection{Dimensión formación práctica}

En este numeral se integran las siguientes dimensiones:

1) contribución de los profesores a la adquisición de la formación práctica, 2) formación práctica recibida por los estudiantes y egresados, 3) importancia que los empleadores le otorgan a la formación práctica, pertinente con el enfoque territorial del desarrollo rural. Tabla 10.

La formación práctica está conformada por 12 ítems, distribuidos en las cuatro dimensiones del enfoque territorial. Los ítems fueron extraídos del currículo oficial. La Tabla 10 presenta los resultados de 8 de los 12 ítems, relacionados con la formación práctica.

Tabla 10. Formación práctica

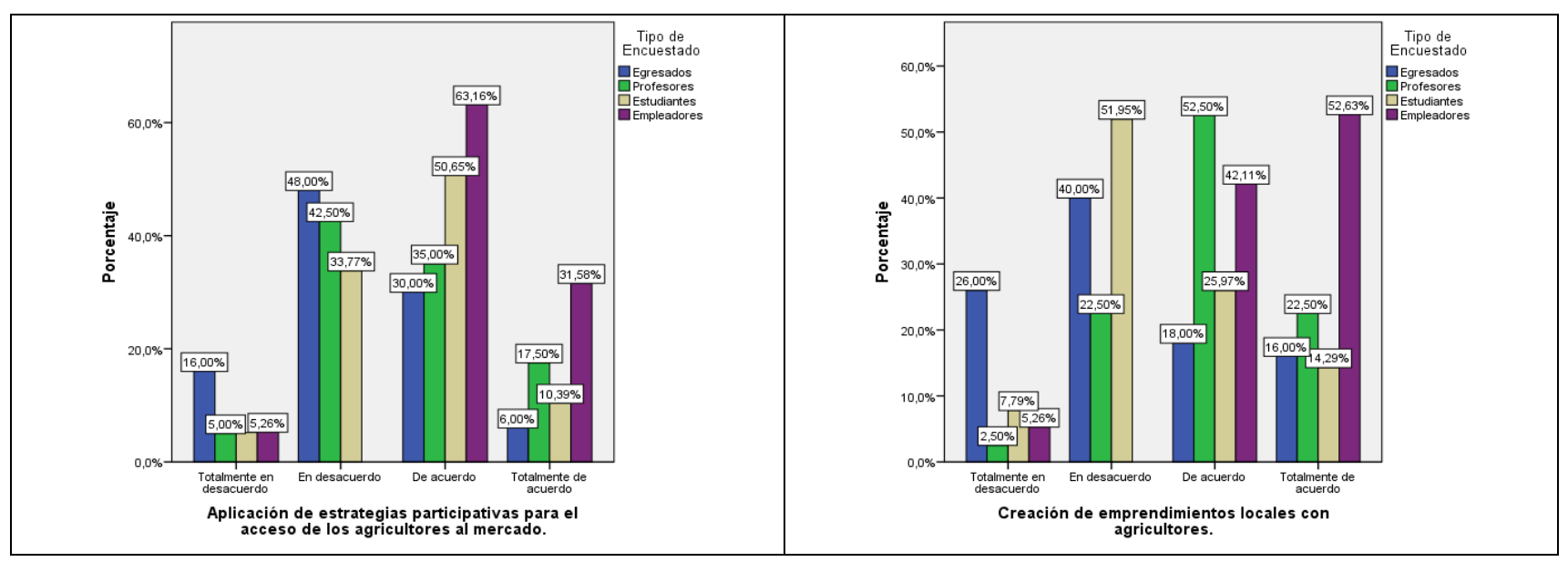




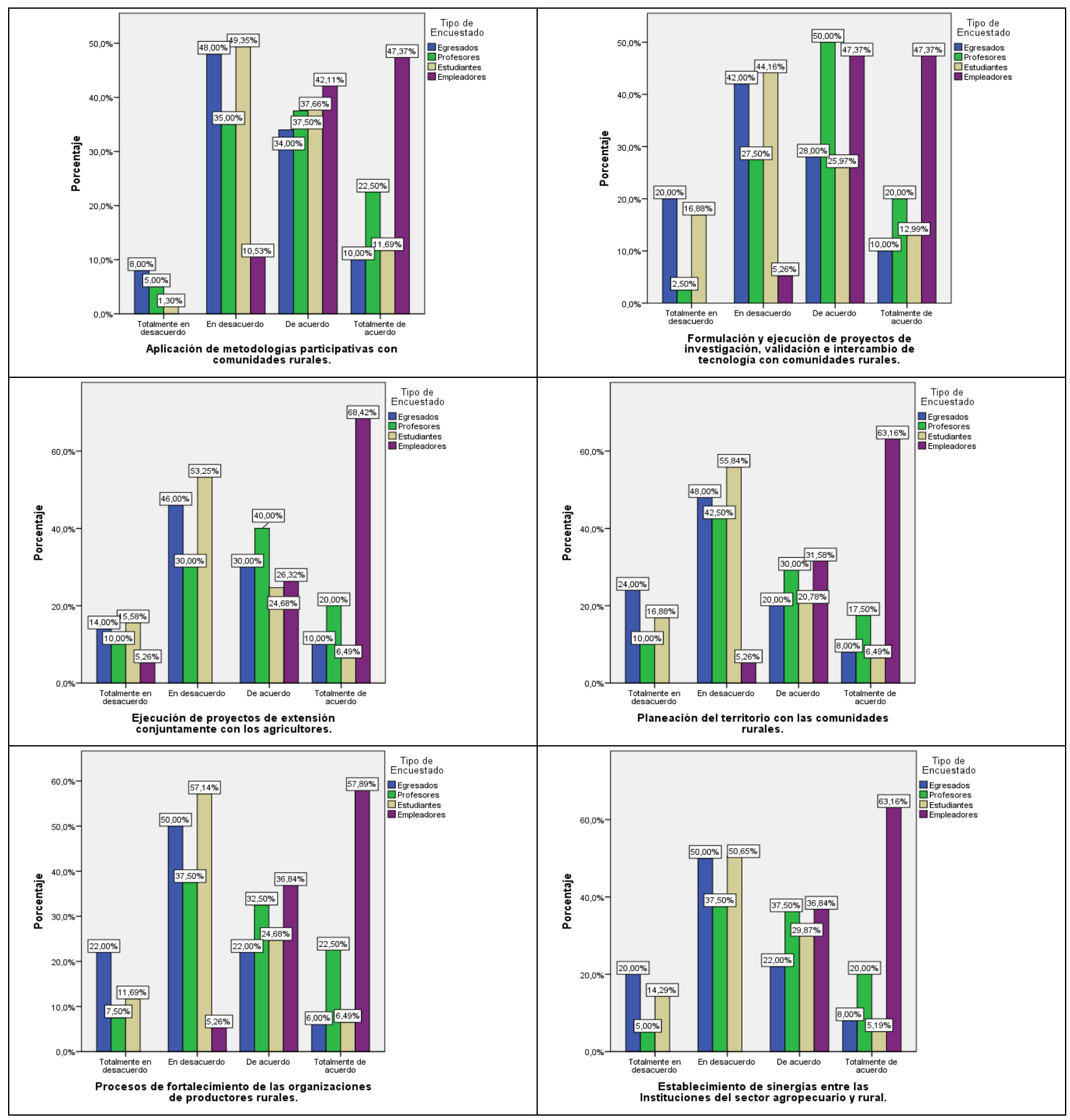

\section{DISCUSIÓN}

El plan de estudios vigente de tecnología agropecuaria, fue formulado en 2004 y pese a que está reacreditado de alta calidad hasta el año 2022, entre 2004 y 2015, no se había realizado oficialmente ningún estudio de prospectiva o de pertinencia social y laboral.

El análisis del currículo, las entrevistas llevadas a cabo con las directivas académicas y la integración de estos datos con los resultados cuantitativos, permiten inferir que en general, la comunidad académica desconoce el modelo de currículo y las perspectivas teóricas en las cuales se fundamenta el programa. El actual modelo de currículo, es funcionalista y aunque el proyecto educativo del programa adhiere teóricamente a perspectivas teóricas constructivistas, desarrollistas o a enfoques psicológicos cognitivos del aprendizaje, las evidencias indican que en la práctica docente, prevalecen modelos pedagógicos heteroestructurantes, tradicionales transmisionistas.

Un análisis detallado de las plantillas y guías didácticas de las asignaturas, reflejó que los 
profesores ( $70 \%$ catedráticos contratados por horas), diligencian estos formatos, sin tener en cuenta el perfil de ingreso de los estudiantes, el modelo pedagógico, el nivel de formación del programa y el perfil de egreso. De hecho, se pudo evidenciar que dos programas diferentes como el de Tecnología e Ingeniería Agropecuaria que pertenecen a la misma Facultad, comparten, en algunos casos, idénticas plantillas del programa y guías didácticas de las asignaturas en casi todas las áreas, es decir, estudiantes pertenecientes a dos programas con diferente nivel de formación, reciben asignaturas conjuntamente, incluyendo las asignaturas del área de formación profesional.

El actual perfil de ingreso muestra que los estudiantes no residen en el campo, no se formaron en instituciones educativas con media técnica en agropecuaria y, dadas las limitaciones económicas y el alto número de docentes catedráticos contratados por horas, son pocas las oportunidades que tienen para interactuar con las comunidades rurales.

Como complemento a lo anterior, las mayores frecuencias de respuesta dadas por los egresados y empleadores, coinciden en hay cinco de los seis cargos declarados en el perfil ocupacional que los tecnólogos no desempeñan, es decir, el encargo social no está claro. La mayor frecuencia de respuesta dada por los egresados y empleadores coincide en que siempre el cargo que desempeña el tecnólogo es el de "asistente técnico", 38\% y 53\% respectivamente. Así mismo, coincidiendo en algunos casos con las mayores frecuencias de respuesta obtenidas de los empleadores, existen siete de las diez funciones, que los egresados manifiestan nunca realizan en su ejercicio laboral.

Las tres funciones que siempre o casi siempre, desempeña el tecnólogo agropecuario y en la cual se presentaron las mayores frecuencias por parte de egresados y empleadores, son dirigir programas de conservación y manejo racional de los recursos naturales renovables; ejecutar programas de instrucción agropecuaria en centros o comunidades; y desarrollar campañas relacionadas con el adelanto del sector campesino. Estos hallazgos se constituyen en un argumento de fondo que justificaba llevar a cabo esta investigación.

El perfil profesional expresa que, la tecnología agropecuaria tiene como propósito formar recurso humano capacitado $[\ldots]$ para mejorar "las condiciones de vida de las comunidades rurales, con criterios de competitividad, desarrollo económico sostenible y equidad social". Se trata de un conjunto de propósitos que reúnen las dimensiones del enfoque territorial. ¿Pero qué muestran los resultados?

El análisis documental indica que las plantillas de los programas y guías didácticas de las asignaturas, incluyen explícitamente competencias transversales, contenidos teóricos y prácticos para responder a estos propósitos.

Cuando se refiere a la formación práctica, las mayores frecuencias de respuesta dadas por los profesores, indican que ellos están de acuerdo en que la práctica docente está contribuyendo con: la creación de emprendimientos locales con los agricultores, con un 52.5\%; formulación y ejecución de proyectos de investigación, validación e intercambio de tecnología con comunidades rurales, con un 50\%; y ejecución de proyectos de extensión conjuntamente con los agricultores, con un $40 \%$. Por el contrario, los egresados y estudiantes se muestran en desacuerdo, así por ejemplo (mayores frecuencias de respuesta): creación de emprendimientos locales con los agricultores, con un $40 \%$ y $51.95 \%$; y formulación y ejecución de proyectos de investigación, validación e intercambio de tecnología con comunidades rurales, con un $42 \%$ y $44.16 \%$, respectivamente, estos dos últimos ítems, tienen que ver con las dimensiones económicoproductiva y socio-cultural, que buscan transformaciones relevantes en la competitividad y la equidad respectivamente, generando dudas acerca de la contribución del programa a la formación integral de sus egresados.

Los altos índices de desempleo, los bajos salarios, el bajo impacto de los egresados en el medio social y académico, los problemas identificados en el perfil de egreso, las complejidades a las cuales se enfrentan los graduados en el medio rural colombiano, sumado, al enfoque territorial como fundamento del desarrollo rural en Colombia, en un escenario de posconflicto, plantean la necesidad de revisar integralmente el diseño curricular del programa y lo que viene ocurriendo en la práctica docente. Esto hace pensar que es necesario transitar hacia un modelo de currículo reflexivo, que contenga una visión territorial multidimensional de la ruralidad, en el marco de pedagogías transformadoras, que permitan hacer un análisis crítico de la realidad, para proponer "acciones educativas y sociales encaminadas a promover la transformación social". (Ayuste y Trilla, 2005, p.224). La pertinencia de estas acciones requiere contar con profesores formados en la ruralidad, con capacidad de llevar a cabo prácticas docentes "basadas en el lugar" (Azano y Stewart, 2015, p.2), que trasciendan a una comprensión 
"compartida del desempeño social, económico y ambiental de los sistemas agrícolas" (Jordan, Wyse y Colombo, 2012, p. 2425-2426) y rompan con el aislamiento y la supremacía de unas áreas sobre las otras, por campos del saber transdisciplinarios, sistémicos. Un docente innovador, capaz de entender el impacto de la investigación rural en relación con los participantes (Burfoot-Rochford, 2015), que derive en una proyección social del programa, visibilizada en el diseño, desarrollo y evaluación del currículo, participativa, con arraigo, contextualizada, con agentes rurales reales, sistematizada y con indicadores sociales.

Este escenario demanda una didáctica con enfoque práctico interpretativo y crítico (SierraArizmendiarreieta y Perez, 2007), aprendiendo en contexto y creando situaciones de aprendizaje vinculadas a la acción-reflexión-acción, en conexión directa con los objetos de trabajo del estudiante y sus campos de acción (Dopico y García-Vasquez, 2011; Jordan, et. al., 2012) y un proceso evaluativo enfocado a la solución a los problemas del medio rural.

\section{CONCLUSIONES}

La integración de los datos obtenidos permite concluir que el diseño, desarrollo y evaluación del currículo no son pertinentes con el enfoque territorial, debido a que los ambientes de aprendizaje donde actualmente están siendo construidos los valores, los conocimientos y las prácticas, están muy alejados del campo y de la realidad que viven sus protagonistas.

El programa debería establecer una relación simbiótica con la ruralidad, de un lado porque el objeto de trabajo y los campos de actuación de los egresados dependen de los recursos naturales, y del otro porque es justamente esa dependencia la que determina que el medio rural se configure en el ambiente de aprendizaje para la práctica situada y se abra la posibilidad para los estudiantes de cualificarse en su complejidad multidimensional.

La práctica docente situada en la ruralidad posibilita el encuentro dialógico, intersubjetivo, entre estudiantes y profesores con los agricultores y otros agentes sociales, actuando en contextos diferenciados como un dispositivo pedagógico mediante el cual se planea, se participa, se investiga, se consolidan emprendimientos locales o se intercambian tecnologías y saberes, haciendo partícipe a la comunidad educativa del proceso de construcción social de los territorios rurales y la acción colectiva contextualizada.

\section{REFERENCIAS}

Acuerdo final. (2016). Acuerdo final para la terminación del conflicto y la construcción de una paz estable y duradera. Recuperado el 20 de Febrero de 2017, de https://www.mesadeconversaciones.com.co/sites/default /files/24-1480106030.11-

1480106030.2016nuevoacuerdofinal-1480106030.pdf

Ambrosio-Albalá, M., y Bastiaensen, J. (2010). The new territorial paradigm of rural development: Theoretical foundations from systems and institutional theories. Institute of Development Policy and Management. Recuperado el 21 de Mayo de 2015, de http://www.ua.ac.be/objs/00251118.pdf

Ayuste, A., y Trilla, J. (2005). Pedagogías de la modernidad y discursos postmodernos sobre la educación. Revista de educación, (336), 219-248.

Azano, A., y Stewart, T. (2015). Exploring place and practicing justice: Preparing pre-service teachers for success in rural schools. Journal of Research in Rural Education, 9(30), 1-12.

Burfoot-Rochford, I. (2015). Book Review. Doing educational research in rural settings: Methodological issues, international perspectives and practical solutions. Journal of Research in Rural Education, 30(11), 1-2.

Cádiz, J., Villanueva, O., Astorga, M., y Echenique, M. (2012). ¿Profesores competentes o humanizadores? Educación y Educadores, 15(3), 535-546.

Casarini, M. (2012). Teoría y diseño curricular. México: Trillas.

Cullen, R., y Hill, R. (2013). Curriculum designed for an equitable pedagogy. Education Sciences, 3(1), 17-29.

Díaz, A. (2005). El docente y los programas escolares. Lo institucional y lo didáctico. Barcelona: Pomares.

Díaz, F. (2003). Main Trends of Curriculum Research In Mexico. En International handbook of curriculum research (págs. 457- 469). Londres: Lawrence Erlbaum Associates, Inc., Publishers.

Dopico, E., y Garcia-Vazquez, E. (2011). Leaving the classroom: A didactic framework for education in environmental sciences. Cultural Studies of Science Education, 6(2), 311-326. doi: http://dx.doi.org/10.1007/s11422-010-9271-9

Galvani, V. (1990). Mirando al 92, el fin de una utopía educativa: el desarrollismo en América Latina. Revista complutense de Educación, 1(13), 477-485.

Gómez, V. (2015). La pirámide de la desigualdad en la educación Superior en Colombia. Diversificación y tipología de instituciones. Bogotá: Universidad Nacional de Colombia.

Grabowski, R. (2008). Modes of long-run development: Latin America and East Asia. Journal of Institutional Economics, 1(4), 25-50. doi:http://dx.doi.org/10.1017/S1744137407000835

Hansen, B., y Greve, A. (2015). The role of human and social capital in dairy farming. Rural Society, 24(2), 154-176. doi: http://dx.doi.org/10.1080/10371656.2015.1060718

Hidalgo, A. (2011). Economía política del desarrollo. La construcción retrospectiva de una especialidad académica. Revista de economía mundial, (28), 279-320.

Hinojo, F., Cáceres, M., y Raso, F. (2013). Análisis de los Componentes Organizativos de Centros de Formación Profesional en España. Revista Latinoamericana de ciencias Sociales, Niñez y Juventud, 11(2), 783-801. doi:10.11600/1692715x.11222180213

Jankovic, D. (2012). Territorial approach to regional rural development. Ekonomika Poljoprivrede, 59(4), 675-686. 
Jordan, N., Wyse, D., y Colombo, B. (2012). Linking agricultural bioscience to cross-sector innovation: A new graduate curriculum. Crop Science, 52(6), 24232431.

Kemmis, S. (1998). El currículum más allá de la teoría de la reproducción. Madrid: Ediciones Morata.

Laanemets, U., y Kalamees, K. (2013). The Taba-Tyler Rationales. Journal of the American Association for the Advancement of Curriculum Studies, 9, 9-12.

Méndez, M. (2008). Integration of socio-humanistic component in the professional training of agricultural education: perceptions, purposes and challenges. Revista Facultad Nacional de Agronomía, 61(2), 4471-4479.

Molina, J. (2010). Keys for rural territorial development. Agronomía Colombiana, 28(3), 429-435.

Murcia, H. (2011). Agribusiness model approach to territorial food development. Agronomía Colombiana, 29(1), 125132.

Ocampo, J. (2014). Misión para la transformación del campo. Saldar la deuda histórica con el campo. Marco conceptual de la misión para la transformación del campo. Bogotá: Departamento Nacional de Planeación. Recuperado el 23 de Mayo de 2015, de https://colaboracion.dnp.gov.co/CDT/Prensa/DOCUME NTO\%20MARCO-MISION.pdf

Pachón, F. (2006). Las concepciones de desarrollo rural en los estudiantes de una facultad de Agronomía en Bogotá (Colombia). Agronomía Colombiana, 24(2), 378-379.

Parr, D., Trexler, C., Khanna, N., y Battisti, B. (2007). Designing sustainable agriculture education: Academics' suggestions for an undergraduate curriculum at a land grant university. Agriculture and Human Values, 24(4), 523-533. doi: 10.1007/s10460-007-9084-y

Pisani, E., y Francescheti, G. (2011). Territorial approaches for rural development in Latin America: a case study in Chile. Revista Facultad de Ciencias Agrarias UNCUYO, (1), 201-208.

Plan Nacional de Desarrollo 2014-2018 (2015). Todos por un nuevo País. Recuperado el 18 de abril de 2017, de https://colaboracion.dnp.gov.co/CDT/PND/PND\%20201 4-2018\%20Tomo\%201\%20internet.pdf

Posner, G. (2004). Análisis del currículo. México: McGrawHill.

Programa de las Naciones Unidas para el Desarrollo PNUD. (2015) ¿Qué es el desarrollo humano? Recuperado el 29 de Junio de 2015, de http://desarrollohumano.org.gt/desarrollohumano/concepto/

Ramo, M. (2011). El nuevo espacio rural en el Salvador. Análisis de un programa educativo de participación comunitaria. Revista de currículum y formación del profesorado, 15(2), 93-107.

Rodríguez, H., y Ramírez, C. (2015). Abordaje metodológico para formulación participativa de planes de asistencia técnica agropecuaria con enfoque territorial. Acta Agronómica, 64(4), 307-315. doi:https://doi.org/10.15446/acag.v64n4.45162

Sánchez, P., Gallardo, R., y Ceña, F. (2016). La noción de resiliencia en el análisis de las dinámicas territoriales rurales: Una aproximación al concepto mediante un enfoque territorial. Cuadernos de Desarrollo Rural, 13(77), 93-116. doi:http://dx.doi.org/10.11144/Javeriana.cdr13-77.nrad

Sierra y Arizmendiarrieta, B., y Pérez, M. (2007). La comprensión de la relación teoría-práctica: una clave epistemológica de la didáctica. Revista de Educación (342), 553-576.
Silva, I., y Sandoval, C. (2012). Metodología para la elaboración de estrategias de desarrollo local. Santiago: Instituto Latinoamericano y del Caribe de Planificación Económica y Social y CEPAL. Recuperado el 12 de Septiembre de 2013, de http://www.eclac.org/publicaciones/xml/6/47546/S76MMetodologia_esp.pdf

Sipos, Y., Battisti, B., y Grimm, K. (2008). Achieving transformative sustainability learning: engaging head, hands and heart. International Journal of Sustainability in Higher Education (IJSHE), 9(1), 68-86. doi: $10.1108 / 14676370810842193$

Thuesen, A., y Nielsen, N. (2014). A Territorial Perspective On Eu'S Leader Approach In Denmark: The Added Value Of Community-Led Local Development Of Rural And Coastal Areas In A Multi-Level Governance Settings. European Countryside, 307-326. doi: 10.2478/euco2014-0017

Wang, N., y Liu, F. (2014). Restrictive factors of vocational education development in cultivation of rural practical skilled personnel and countermeasures. Asian Agricultural Research, 6(6), 70-73. 